\title{
Analysis of Stakeholder Benefits of NextGen Trajectory-Based Operations
}

\author{
Guillermo Calderón-Meza (Ph.D. Candidate) \\ Research Assistant \\ Center for Air Transportation Systems Research \\ George Mason University \\ Fairfax, Virginia, 22030, USA \\ Email: gcaldero@gmu.edu
}

\author{
Lance Sherry (Ph.D) \\ Director \\ Center for Air Transportation Systems Research \\ George Mason University \\ Fairfax, Virginia, 22030, USA \\ Email: 1sherry@gmu.edu
}

\begin{abstract}
Trajectory-Based Operations is a NextGen initiative that seeks to increase the effective-capacity of the National Airspace System by adding flexibility to the 4-D trajectories as flights traverse airspace. More flexible trajectories can minimize the airlines operating costs (i.e., distance flown), shift the geography of Air Traffic Control (ATC) workload (i.e., sectors used), shift the time-intensity of ATC workload (i.e., flights counts per sector), and reduce congestion in the destination airports.

This paper describes the results of an analysis of one day of operations, including domestic and international flights, in the NAS using traditional navigation aid-based airway routes compared to direct, i.e., Great Circle Distance, routes. Apart from the total of 1,608,751 $\mathrm{nm}$ (average $24.7 \mathrm{~nm}$ per flight) savings in distance flown generated by flying Great Circle Distance Routes, the results show: (i) a redistribution of flights across sectors resulting in a reduction of $3 \%$ in the total time the flights in a sector are in excess of the Monitor Alert Parameters for that sector, (ii) a reduction in ATC workload reflected by a $42 \%$ drop in the number of flights requiring conflict resolution, (iii) the average ground delay due to the natural limits in the arrival capacity of the OEP-35 airports increases when using direct routes, but the increase is small, (iv) the congestion at the destination airports remains the same. These results indicate the upper bound of benefit opportunities for both ATC and the airlines based on the introduction of flexible routing structures in NextGen, but no improvement for the airports in terms of congestion.
\end{abstract}

Index Terms-NextGen, evaluation, conflicts, FACET, distance flown, delays.

\section{INTRODUCTION}

NextGen [1] enabling technologies and operational initiatives seek to increase the effective-capacity of the National Airspace System (NAS) by opening up unused airspace, increasing the availability of airspace in all weather conditions, and increasing the utilization of existing airspace by reducing spacing between flights on the same routes.

Concepts of operations, such as Trajectory-based Operations (TBO) [1], will allow flights increased flexibility in their 4-D trajectories as they traverse center airspace.

Trajectory-Based Operations is a NextGen initiative that adds flexibility to the 4-D trajectories of flights as they traverse airspace. More flexible trajectories can minimize the airlines operating costs by reducing distance flown. The changes in the trajectories will shift the geographic distribution of flights across Air Traffic Control (ATC) sectors, as well as the distribution of instantaneous flight counts in individual sectors. More flexible routes will not reduce congestion at the destination airports since flights still must arrive to the same destinations regardless of the type of route they use. If flights take longer or shorter routes to modify their actual arrival times, then the distribution at destination airports will change in time. This shift in time could reduce congestion, but the results show that this is not the case.

The Trajectory-Based Operations ideas can be applied to three types of operations in the NAS [1], [2]. 1) The arrival and departure operations. These operations include Continuous Decent Arrivals (CDA) that smooth the transition from top-ofdecent to near idle speed. And Tailored Arrivals that use automation and data communication technology to provide a preferred trajectory path and transfer it to the flight management system on the aircraft. 2) The en route and cruise operations. These operations include 3D Path Arrival Management that designs fuel-efficient routes to decrease controller and pilot workloads. The 4D Trajectory-Based Management that defines 3-dimensional flight paths based on points in time (the 4-D) from gate-to-gate. And the Required Navigation Performance in which navigation performance requirements for operation within an airspace define the trajectories. In this paper, only the 3D Path Arrival Management and some of the 4D Trajectory Managements are considered.

This paper extends the analysis of a previous paper [3] for the potential upper limit of the impact of the shifting trajectories to minimize the airlines operating costs (i.e., less fuel burn due to less distance flown), the geographic workload (i.e., sectors used), the time workload (i.e., flights counts per sector) for Air Traffic Control (ATC), and the congestion (i.e., number of arrival per unit of time) at the destination airports. The previous paper used a single day of only US domestic flights, e.i., lower demand than it is in reality. The congestion at the destination airports was not considered in the previous study either. Similar studies have been carried out to evaluate the impact of this change and other changes proposed by NextGen. Barnett [4] evaluates the impact in 
safety caused by using direct routes instead of airways. The study concludes that using direct routes diminish the risk of enroute collision. These results are valid only if certain rules for TFM remain in effect after the change. A caveat of the study is that the results will depend on the capacity of the technology and humans to match the current performance of the ATC. Agogino and Tumer [5] evaluate policies intended to optimize performance of the TFM. The metrics used are congestion and delays. The study evaluates several ATC algorithms as well as the use of multi-agent technology. The algorithms achieve significant improvements in performance compared to previous algorithms and the current practices. Magill [6] also analyzes the change from airway routes to direct routes. The study uses the number of conflicts (called interactions in this case) as metric for the ATC workload. The study modifies the separation rules as well as the type of routes. The paper concludes that the reduction of traffic density due to the use of direct routes is the most significant factor in the reduction of workload for the ATC.

The Future ATM Concept Evaluation Tool (FACET ${ }^{1}$ ) [7] was used for this experiment. The input data consisted of 65,173 flights in the US including domestic and international traffic. A total of 287 US airports (4,170 US O/D pairs). The experiment had two scenarios: (i) flights followed Great Circle Distance (GCD) routes from TRACON to TRACON, and (ii) flights followed traditional navigation aid-based airway routes. The flights in each scenario used the same cruise flight levels and cruise speeds. An expected result from the use of Great Circle Distance from TRACON to TRACON is a reduction in the total distance flown. This is because the land-based navigation aids used today to give structure to the airspace are not always on a Great Circle Distance between any two TRACONs. The results, including the reduction in flown distance, are summarized below:

(i) Great Circle Distance routes generated a total of $1,608,751 \mathrm{~nm}$ (average $24.7 \mathrm{~nm}$ per flight) savings in reduced distance flown.

(ii) Great Circle Distance routes resulted in a redistribution of ATC workload reducing the time sectors were above their Monitor Alert Threshold (MAP) from 35\% to 32\% of the total work time.

(iii) Great Circle Distance routes resulted in reduced ATC workload reducing the number of detected conflicts by $42 \%$.

(iv) The average ground delay due to the natural limits in the capacity (i.e., VFR conditions) of the OEP-35 airports increases only 24 seconds when using direct routes. This is increase is small enough to be disregarded.

(v) The congestion at the destination airports reduces an average of 0.19 arrivals per 15 minute bin when using direct routes. Though this reduction is statistically significant, i.e., the $95 \%$ confidence interval is $[0.17,0.20]$, it is smaller than 1 . So it can be concluded that there is no change in the congestion at the destination airports.

${ }^{1}$ See www.aviationsystemsdivision.arc.nasa.gov/research/modeling/facet.shtml
These results establish an upper limit on the benefits of using Trajectory-Based Operations in the form of 3D Trajectory Management. The result is a win-win scenario for both the airlines and air traffic control. The use of Great Circle Distance routes geographically redistributed the flights reducing workload in the sectors and well as significantly reducing conflicts in flight trajectories. It should also be noted that the use of Great Circle Distance routes did not alleviate the flight delays resulting from over-scheduled departure and arrivals.I do not really show this in the paper, or I am not enumerating this in the results

This paper is organized as follows: Section 2 describes the method and the design of the experiment, the simulation used for the experiment, and the configuration and parameters used in the experiment, Section 3 describes the results of the experiment, and Section 4 provides conclusions, implications of these results, and future work.

\section{Method And Design of the Experiment}

The experiment was conducted using the Future ATM Concept Evaluation Tool (FACET) [7]. The tool has been used in previous studies [3], [5], [8], [9] to evaluate new Traffic Flow Management (TFM) concepts in the NAS. FACET offers many options like the possibility connecting to real-time data sources for weather and traffic, real-time conflict detection and resolution, batch processing of input data, and a Java $\mathrm{API}^{2}$. In the absences of random inputs, e.g., weather phenomena, the simulation is deterministic. The results will be the same regardless of the number of executions.

Many outcomes of the simulation like number of sectors or centers flown, distance flown, origin and destination airports, distance flown, take off and landing times, delays due to Ground Delay Programs, and number of conflicts, can be obtained from the API or from the GUI ${ }^{3}$.

\section{A. The input files for FACET}

In this experiment, the input data for FACET was a tracking data file (called TRX by FACET). The file was obtained from a collaborator and it represents historical data of the real demand for a period of about 35 days.

Tracking data are not suitable for this type of experiment, since the goal is to simulate the behavior of the flights when given different rules. The real need for this experiment is a schedule of the all the flights in the NAS, plus cruise speeds (to estimate arrival times) and altitudes (to reflect the type of aircraft used). So the original data file was filtered to obtain only a single record per flight. The record contains the following data: 1) the time in which each flight enters the simulation (usually the takeoff time), 2) the aircraft id or flight number, 3) the aircraft type (required to determine its performance profile), 4) the initial coordinates when it enters the simulation, 5) the initial altitude (the flight could enter the simulation when it is already flying), 6) the initial heading of the aircraft, 7) the commanded speed, 8) the current center and

\footnotetext{
${ }^{2}$ API: Application Program Interface.
}

${ }^{3}$ GUI: Graphical User Interface. 
sector (for US flight only), 9) the filed altitude, and 10) the flight plan string. The filtering process was done by a parser written in the Java programming language for that purpose.

A TRX file generated this way does not track the flights through the National Airspace System, it only describes every flight with a single record. So this file can be used for simulation purposes in FACET only, not for playback.

The TRX file used in this experiment contains 65,173 domestic (USA) and international flights included in the traffic in the NAS from August 172006 at 00:02:00 GMT to August 192006 at 18:03:00 GMT.

\section{B. Design of Experiment}

The goal of this experiment is to evaluate the effect of applying TBOs to the en route flights. Specifically, flights change from using airway routes, i.e., flight plans consisting of sequences of land-based navigation aids, to Great Circle Distance routes from TRACON to TRACON, i.e., direct routes.

This paper presents and compares the results of one experiment divided into two scenarios (see Table IV). The first scenario simulates one day of NAS operations in which all the flights use navigation aid-based airway routes, i.e., flight plans, as it is done today in the NAS. The second scenario simulates the same day of operations, but flights follow Great Circle Distances routes, i.e., direct routes, from TRACON to TRACON.

The outcomes of interest for each scenario are the total distance flown by the flights, the total number of conflicts detected, the flight delays generated in the OEP- $35^{4}$ airports, and the number of arrival per unit of time at the destination airports. The benefits for the airlines, controllers, and airports can be determined using these outcomes.

FACET uses the same input file in both scenarios, so each flight in one scenario has a corresponding flight in the other scenario. So individual data like distance flown and flight delay can be compared using a paired two-tail t-test to determine the effect of the change. For still not understood reasons, some flights do not appear in both scenarios. Only flights that appear in both scenarios are used in the t-test.

The comparison of the total number of conflicts is only done for a single pair of numbers, so no statistical test is applied in this case.

The distribution of the sectors load is multi-dimensional. There is spatial distribution and temporal distribution. In this paper only the temporal distribution will be analyzed, leaving the spatial distribution for future work. The two scenarios are compared using the percentage of time in which, at least, one sector contains a number of flights that is on or over the sectors Monitor Alert Parameter (MAP) value, i.e., it is over-loaded. To get an idea of the distribution, also the percentage of time in which at least one sector is at or over $80 \%$ of its MAP is compared between scenarios.

No external disturbances are included during the simulations, i.e., there are no restrictions due to weather, congestion,

\footnotetext{
${ }^{4}$ OEP: Operational Evolution Partnership Plan.
}

push-back delays, or other stochastic events. So, the simulations are deterministic. The only limitation that is imposed in the arrival capacity of the EOP-35 airports, which is set to the VFR departure and arrival rates for the whole day (see Table I). Even with VFR rates, this limitation generates ground delays via Ground Delay Programs (GDP). The use of these GDPs adds some time constraints to the trajectories of the flights. This is the only $4 \mathrm{D}$ aspect of TBO that is included in this paper. The ground delays are compared using the total minutes of delays and the average minutes in the OEP-35 airports which are the only ones restricted using GDPs.

\section{FACET settings used in experiment}

In this experiment, FACET takes its input from batch files, and the outputs are taken from the simulation via the API. The input file was loaded using the loadDirectRouteSimAsynch and the loadFlightPlanSimAsynch functions of the API. With the first function, FACET sets itself to use Great Circle Distance routes, i.e., direct routes. With the second function, FACET uses the airways routes, i.e., flight plans, provided in the input file. Both functions accept the same number and types of arguments. The trajectory update interval is set to 60 . The integration time step is set to 60.0. And the additional update delay is set to 0.2 .

The API provides an interface (ConflictInterface) with functions to enable (setEnabled) and configure (setConflictDetectionParameters) the conflict detection functionality. The parameters are as follows. The center index is set to -1 , i.e., all the centers. The surveillance zone is $120 \mathrm{~nm}$. The lookahead time is 0 . The horizontal separation is $6 \mathrm{~nm}$. The vertical separation below f1290 is $1000 \mathrm{ft}$. The vertical separation above $\mathrm{f} 1290$ is $1000 \mathrm{ft}$. Also, the detected conflicts are displayed during the simulation.

The arrival rates (AAR) of the airports are infinite by default in FACET. For this experiment, the capacities are limited using FACET's GDP functionality. The OEP-35 airports are assigned a maximum capacity in the form of an arrival delay GDP from the 0:00 to 24:00 (see Table I). With these limits in the capacity of the airports, FACET starts recording delay statistics during the simulations.

A total of 66.27 hours $(3,976$ minutes $)$ of operations are simulated. All other parameters of FACET are left to their default values.

An external Java program, using the FACET API, measures the distance traveled as follows. At each simulation time step (one minute) the distance flown by each flight is updated based on the previous and current coordinates. The computation of distance is done with the utils.getGCDistanceNM function of FACETs API. The external program also records the total number of flights in each sector, including all the sector levels, i.e., low, high, and super. Distances and sector loads are written into text files for further analyses. 
TABLE I

DEFAULT VFR AIRPORT ARRIVAL RATES (AAR) FOR THE OEP-35 AIRPORTS USED IN THE SIMULATION

\begin{tabular}{|l|c|l|c|}
\hline $\begin{array}{c}\text { Airport } \\
\text { name } \\
\text { (ICAO) }\end{array}$ & $\begin{array}{c}\text { Airport Arrival } \\
\text { Rate } \\
\text { (Moves per hour) }\end{array}$ & $\begin{array}{c}\text { Airport } \\
\text { name } \\
\text { (ICAO) }\end{array}$ & $\begin{array}{c}\text { Airport Arrival } \\
\text { Rate } \\
\text { (Moves per hour) }\end{array}$ \\
\hline KATL & 80 & KLGA & 40 \\
\hline KBOS & 60 & KMCO & 52 \\
\hline KBWI & 40 & KMDW & 32 \\
\hline KCLE & 40 & KMEM & 80 \\
\hline KCLT & 60 & KMIA & 68 \\
\hline KCVG & 72 & KMSP & 52 \\
\hline KDCA & 44 & KORD & 80 \\
\hline KDEN & 120 & KPDX & 36 \\
\hline KDFW & 120 & KPHL & 52 \\
\hline KDTW & 60 & KPHX & 72 \\
\hline KEWR & 40 & KPIT & 28 \\
\hline KFLL & 44 & KSAN & 36 \\
\hline KHNL & 40 & KSEA & 72 \\
\hline KAID & 64 & KIAH & 44 \\
\hline KSFO & 60 & KJFK & 52 \\
\hline KSLC & 44 & KLAS & 84 \\
\hline KSTL & 52 & KLAX & \\
\hline KTPA & 28 & & \\
\hline
\end{tabular}

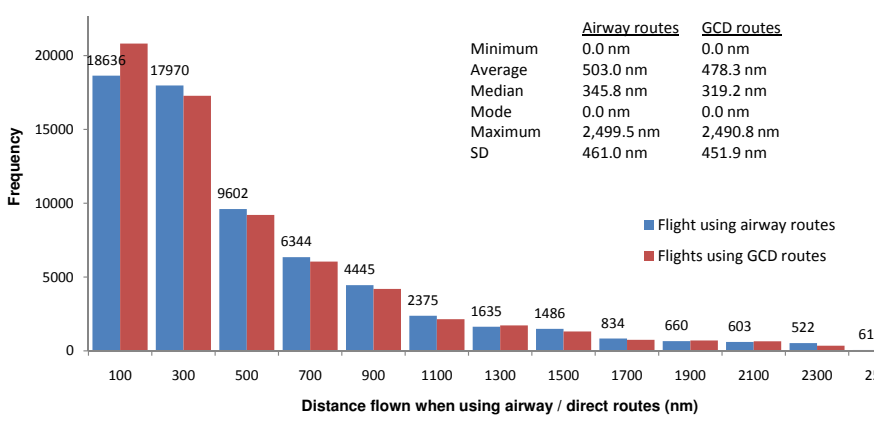

Fig. 1. Histogram of the distances flown when using airway routes and direct routes

\section{RESULTS}

\section{A. Distances flown}

Figure 1 shows the distribution of the distance flown by all the flights using airway routes. The figure also includes the descriptive statistics for this scenario. Most of the flights travel less than $1,000 \mathrm{~nm}$, with a peak of flights between 200 and $400 \mathrm{~nm}$. Short flights, i.e., less than $200 \mathrm{~nm}$ are relatively low in frequency in this input file. There is a long tail in the distribution, but the actual number of flights is low compared to the other distances.

Figure 1 shows the distribution of the distance flown by all flights using direct routes. The figure also includes the descriptive statistics for this scenario. Most of the flights travel less than $1,000 \mathrm{~nm}$, with a peak of flights between 200 and $400 \mathrm{~nm}$. Short flights, i.e., less than $200 \mathrm{~nm}$ are moderate in frequency. There is a long tail in the distribution, but the actual number of flights in the tail is low compared to the other distances.

There are changes in the descriptive statistics with respect to the results of the other scenario. The minimum, average, median, and the standard deviation are smaller in this scenario

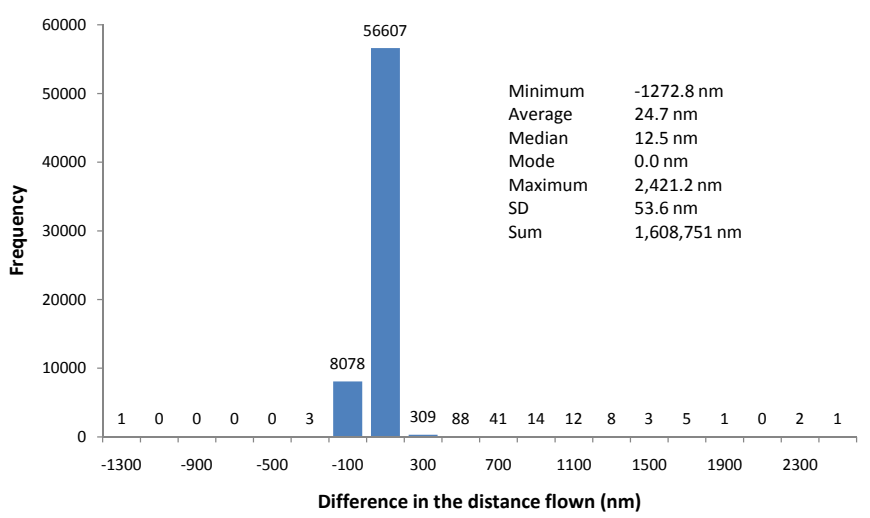

Fig. 2. Histogram of the difference in the distance flown

TABLE II

NUMBER OF MINUTES WITH AT LEAST ONE SECTOR SATURATED OR ON THE VERGE OF SATURATION

\begin{tabular}{|l|c|c|}
\hline \multirow{2}{*}{ Scenario } & $\begin{array}{c}\text { Number of minutes with at least } \\
\text { one sector on or above } \\
\text { of the total } \mathbf{2 , 1 1 4} \text { minutes })\end{array}$ \\
\cline { 2 - 3 } & MAP & $\mathbf{8 0 \%}$ of MAP \\
\hline Flights using airway routes & $1407(35 \%)$ & $1657(42 \%)$ \\
\hline Flights using direct routes & $1284(32 \%)$ & $1595(40 \%)$ \\
\hline
\end{tabular}

than they are in the other scenario. But the mode is greater reflecting the geographical structure of the O/D pairs in the NAS.

Figure 2 shows the distribution of the differences of distance flown by corresponding flights in both scenarios, i.e., it is a paired comparison of distances. The figure also includes the descriptive statistics for the distribution.

A paired two-tail t-test shows that the mean of the difference between the distances flown by corresponding flights in the two scenarios is significantly different than zero $(\mathrm{M}=24.7, \mathrm{SD}$ $=53.6, \mathrm{~N}=65,172), \mathrm{t}=117.6$ and the two-tail $\mathrm{p}=0.000 . \mathrm{A}$ $95 \%$ confidence interval about the mean is $(24.3,25.1)$. This reduction in the average distance flown is a benefit, for the airlines and the environment, of using direct routes. The benefit comes from a reduction in fuel burned, i.e., less pollution and lower costs.

\section{B. Sectors over MAP}

A metric for the load of sectors is a function of time, space, the number of flights, and routes of the flights. The number of flights did not change between scenarios in this experiment. The routes are expected to change significantly when going from flight plans to direct routes. With this change in the type of route the distribution of sector load through time and space is also expected to change.

The time distribution of the sector load is analyzed in this experiment. TABLE II shows that controllers spend $35 \%$ of their time managing congested sectors, i.e., at or above the sector's MAP, when the flights use airway routes. But controllers spend $32 \%$ of their time managing congested sectors when the flights use direct routes. The values for $80 \%$ of MAP give an idea of the distribution of sector load in the two scenarios. 


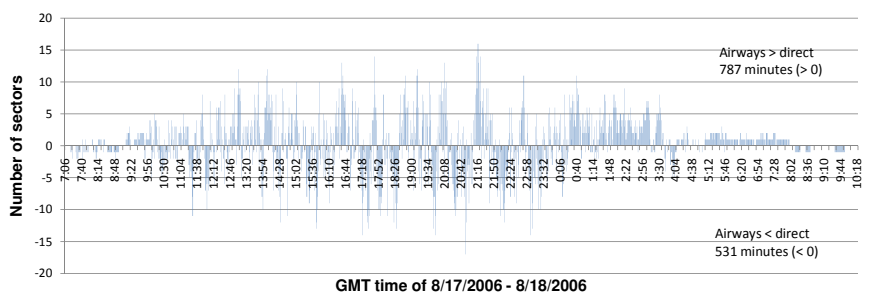

Fig. 3. Minute by minute difference in the number of sectors on or above MAP

The percentage of time controllers spend managing sectors with $80 \%$ or more of their MAPs is similar in both scenarios with a small reduction for the case of direct routes. This similarity indicates that using direct routes mostly reduces the frequency of overloaded sectors, but does not change the total time controllers spend managing "almost saturated" sectors.

Comparing the sector loads minute by minute provides more insight of effect of using direct routes in the NAS. Figure 3 shows that using airways produces load peaks. In 787 minutes of the total simulation time (20\%) the peaks are higher when using airways than when using direct routes (positive side of the vertical axis). But, only in 531 minutes of the total simulation time (13\%), using direct routes produces peaks that are higher than when using airways. The difference of $7 \%$ in the time and a visual inspection of the chart indicate that the peaks are more scattered in time when using direct routes. So controllers will have more time to "rest" between peaks of saturation when flights use direct routes and the saturation will be, in average smaller than when using airways.

\section{Conflicts}

The total number of conflicts detected reduced from 43,894 when using flight plans to 25,638 when using direct routes. This is an improvement in safety, i.e., lower probability of accident, and a further reduction in the workload of the controllers, i.e., they have to resolve $41.6 \%$ less conflicts. Magill [6] found that, for similar separation rules, the reduction was about $35 \%$.

\section{Delays}

The flight ground delays generated by the GDPs defined for the OEP-35 airports are summarized in Table III. The arrival capacities of the OEP-35 airports were set to VFR rates for the whole day. Ground Delay Programs were activated at all the OEP-35 airports.

The total ground delay generated for the OEP-35 airports increases from 107,776.1 minutes when using airway routes to $116,674.9$ minutes when using direct routes. The direct comparison of these two numbers is not possible because the total number of flights arriving at the OEP-35 airports is not the same between scenarios. In the case of the airways 16,589 flights arrived at the OEP-35 airports and were part of the GDPs defined there. In the case of the direct routes, 16,854 flights arrived at the airports and took part in the GDPs. The reason for this difference is still not clear, but it could be the
TABLE III

FLIGHT DELAYS ON THE OEP-35 AIRPORTS OBTAINED BY LIMITING ARRIVAL CAPACITY

\begin{tabular}{|l|r|r|r|r|r|r|}
\hline \multirow{2}{*}{$\begin{array}{c}\text { Airport } \\
\text { code } \\
\text { (ICAO) }\end{array}$} & $\begin{array}{c}\text { Number } \\
\text { of } \\
\text { flights }\end{array}$ & $\begin{array}{c}\text { Total } \\
\text { delay } \\
\text { (min) }\end{array}$ & $\begin{array}{c}\text { Avg } \\
\text { delay } \\
\text { (min) }\end{array}$ & $\begin{array}{c}\text { Number } \\
\text { of } \\
\text { flights }\end{array}$ & $\begin{array}{c}\text { Total } \\
\text { delay } \\
\text { (min) }\end{array}$ & $\begin{array}{c}\text { Avg } \\
\text { delay } \\
\text { (min) }\end{array}$ \\
\hline KATL & 1,208 & $38,589.1$ & 31.9 & 1,232 & $41,445.2$ & 33.6 \\
\hline KBOS & 483 & 543.0 & 1.1 & 489 & 506.8 & 1.0 \\
\hline KBWI & 333 & 688.5 & 2.1 & 340 & 645.2 & 1.9 \\
\hline KCLE & 329 & $1,625.83$ & 4.9 & 332 & $1,759.8$ & 5.3 \\
\hline KCLT & 595 & $3,067.8$ & 5.2 & 603 & $3,163.3$ & 5.2 \\
\hline KCVG & 475 & 700.5 & 1.5 & 477 & 772.2 & 1.6 \\
\hline KDCA & 339 & 606.6 & 1.8 & 345 & 573.1 & 1.6 \\
\hline KDEN & 667 & 330.5 & 0.5 & 678 & 464.0 & 0.7 \\
\hline KDFW & 812 & 403.7 & 0.5 & 820 & 366.2 & 0.4 \\
\hline KDTW & 599 & $2,554.7$ & 4.2 & 617 & $3,374.5$ & 5.5 \\
\hline KEWR & 524 & $5,652.8$ & 10.8 & 532 & $6,070.3$ & 11.4 \\
\hline KFLL & 292 & 440.5 & 1.5 & 294 & 430.2 & 1.4 \\
\hline KHNL & 114 & 106.2 & 0.9 & 115 & 109.0 & 0.9 \\
\hline KIAD & 468 & $1,067.2$ & 2.3 & 479 & $1,440.3$ & 3.0 \\
\hline KIAH & 746 & $4,174.3$ & 5.6 & 755 & $4,909.3$ & 6.5 \\
\hline KJFK & 446 & $2,350.8$ & 5.3 & 455 & $2,686.4$ & 5.9 \\
\hline KLAS & 413 & $1,092.1$ & 2.6 & 424 & $1,322.5$ & 3.1 \\
\hline KLAX & 561 & 431.5 & 0.8 & 569 & 447.9 & 0.8 \\
\hline KLGA & 508 & $3,519.8$ & 6.9 & 513 & $4,026.0$ & 7.8 \\
\hline KMCO & 385 & 498.6 & 1.3 & 388 & 493.3 & 1.3 \\
\hline KMDW & 324 & $1,086.9$ & 3.4 & 329 & $1,057.4$ & 3.2 \\
\hline KMEM & 423 & 653.4 & 1.5 & 428 & 657.0 & 1.5 \\
\hline KMIA & 414 & 334.3 & 0.8 & 429 & 366.4 & 0.8 \\
\hline KMSP & 544 & $2,207.5$ & 4.0 & 549 & $2,931.3$ & 5.3 \\
\hline KORD & 1,144 & $20,881.8$ & 18.2 & 1,159 & $22,390.5$ & 19.3 \\
\hline KPDX & 230 & 646.0 & 2.8 & 239 & 386.3 & 1.6 \\
\hline KPHL & 624 & $4,056.6$ & 6.5 & 625 & $3,553.4$ & 5.7 \\
\hline KPHX & 493 & 966.5 & 2.0 & 503 & $1,277.7$ & 2.5 \\
\hline KPIT & 283 & 143.8 & 0.5 & 290 & 136.4 & 0.5 \\
\hline KSAN & 199 & 436.2 & 2.2 & 200 & 528.1 & 2.6 \\
\hline KSEA & 333 & $1,614.0$ & 4.8 & 337 & $1,743.5$ & 5.2 \\
\hline KSFO & 309 & 263.8 & 0.8 & 324 & 278.7 & 0.8 \\
\hline KSLC & 394 & $4,829.0$ & 12.2 & 402 & $5,156.8$ & 12.8 \\
\hline KSTL & 317 & 310.0 & 1.0 & 319 & 313.0 & 1.0 \\
\hline KTPA & 261 & 902.4 & 3.4 & 264 & 893.0 & 3.4 \\
\hline Totals & $\mathbf{1 6 , 5 8 9}$ & $\mathbf{1 0 7 , 7 7 6 . 1}$ & $\mathbf{6 . 5}$ & $\mathbf{1 6 , 8 5 4}$ & $\mathbf{1 1 6 , 6 7 4 . 9}$ & $\mathbf{6 . 9}$ \\
\hline & & & & & & \\
\hline
\end{tabular}

fact that the other flights did not take part of the GDPs and were not recorded in the results.

The global average of ground delay, i.e. average of the averages for the OEP-35, is 6.5 for the case of the airways and 6.9 for the direct routes. The mean flight delay differs from airport to airport ranging from $31.9 \mathrm{~min}$ to $0.5 \mathrm{~min}$ in the case of the airway routes, but from $33.6 \mathrm{~min}$ to $0.4 \mathrm{~min}$ in the case of direct routes.

These numbers indicate that using direct routing increases the global average ground delay due GDPs by some seconds. This might be caused by the change in the arrival pattern resulting from the shorter distances flown (i.e. presumably shorter flight times also). Since some arrivals will happen earlier than when using airways some modifications in the arrival sequences might be necessary. These modifications would be achieved by the GDPs in place, and will result in small increments in the ground delays. But a more detailed study of the congestion at the arrival airports will give more insight in this issue. 


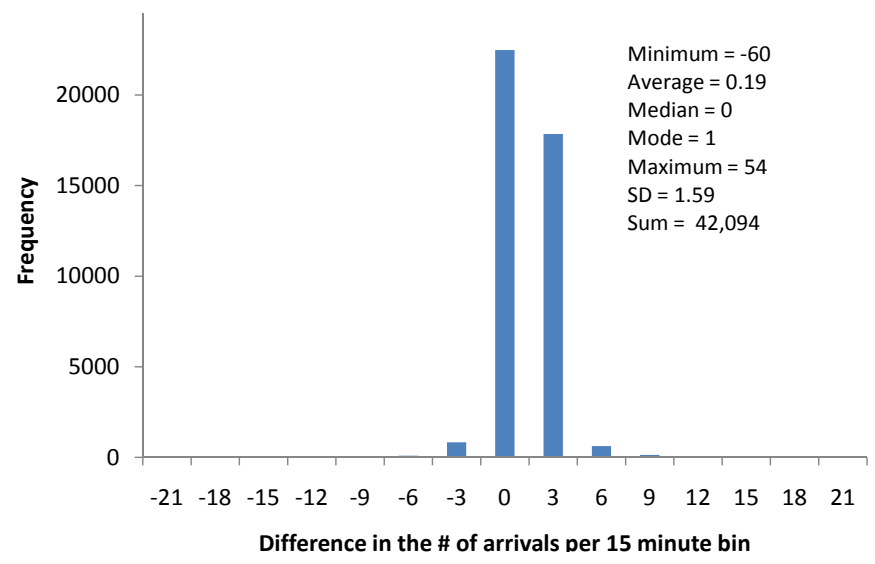

Fig. 4. Histogram of the difference between scenarios in the of arrivals per 15 minute periods for all airports from 8/17/2006 0:02 to 8/19/2006 18:03 GMT

\section{E. Congestion at destination airports}

Figure 4 shows the distribution of the differences of the of arrivals per 15 minute bin for all the airports from 8/17/2006 0:02 GMT to 8/19/2006 18:03 GMT, i.e., the whole simulated time. Corresponding bins are compared so that the difference is arrivals in the 15 minute bin when using airways minus arrivals in the same 15 minute bin when using direct routes, i.e., it is a paired comparison of distances. The figure also includes the descriptive statistics for the distribution.

A paired two-tail t-test shows that the mean of the difference between the number of arrivals at the destination airports for corresponding periods of 15 minutes in the two scenarios is significantly different than zero $(\mathrm{M}=0.19, \mathrm{SD}=1.59, \mathrm{~N}$ $=42,094), \mathrm{t}=24.29$ and the two-tail $\mathrm{p}=0.000$. A $95 \%$ confidence interval about the mean is $(0.17,0.20)$. Since the difference is taken as of arrivals for airways - arrivals for direct routes, the result of the $t$-test indicates a small reduction in the number of arrivals per 15 minutes period. So using direct routes tends to better distribute the arrivals in time effectively reducing congestion at the destination airports. But, the reduction is small in general.

The average of arrivals per 15 minute bin for all the airports from 8/17/2006 0:02 GMT to 8/19/2006 18:03 GMT when airways is 1.61 arrivals/15min ( $\mathrm{SD}=2.58)$. When using direct routes, the average is 1.42 arrivals $/ 15 \mathrm{~min}(\mathrm{SD}=2.61)$. The average is smaller when using direct routes, but the standard deviation is greater. This is an indication of a wider, but shorter distribution in the case of the direct routes, i.e., the arrivals are more scattered in time.

TABLE IV summarizes the results of the two scenarios and the previous tables and charts.

\section{CONCLUSIONS}

This experiment consisted of two scenarios with the same set of 19,318 domestic flights in the NAS. The scenarios were executed using FACET. In one scenario flights used airways the same way they currently do in the NAS. In the second scenario flights used direct routes. The arrival rate of the
TABLE IV

SUMMARY OF THE RESULTS

\begin{tabular}{|c|c|c|}
\hline & \multicolumn{2}{|c|}{ Scenario } \\
\hline & $\begin{array}{c}\text { Great Circle } \\
\text { Distance routes }\end{array}$ & Airway routes \\
\hline $\begin{array}{l}\text { Total distance flown } \\
\text { (Average distance per } \\
\text { flight) }\end{array}$ & $\begin{array}{l}12,668,412.1 \mathrm{~nm} \\
(655.8 \mathrm{~nm})\end{array}$ & $\begin{array}{c}13,236,239.4 \mathrm{~nm} \\
(685.2 \mathrm{~nm})\end{array}$ \\
\hline $\begin{array}{l}\text { Percentage of time } \\
\text { with sectors above } \\
\text { MAP threshold (\% of } \\
\text { time with sectors } 80 \% \\
\text { or more of MAP) }\end{array}$ & $32 \%(40 \%)$ & $35 \%(42 \%)$ \\
\hline $\begin{array}{l}\text { Number of airborne } \\
\text { conflicts detected by } \\
\text { ATC }\end{array}$ & 25,638 & 43,894 \\
\hline $\begin{array}{l}\text { Total flight delays } \\
\text { (Average delays) }\end{array}$ & $\begin{array}{l}20,916.25 \mathrm{~min} \\
(2.28 \mathrm{~min})\end{array}$ & $\begin{array}{l}21,396.53 \mathrm{~min} \\
(2.26 \mathrm{~min})\end{array}$ \\
\hline 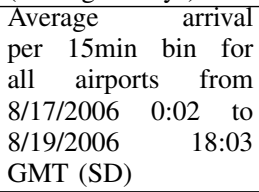 & $1.42(2.61)$ & $1.61(2.58)$ \\
\hline
\end{tabular}

OEP-35 airports was set to the VFR rates using the GDP functionality provided by FACET.

The goal of the experiment was to evaluate the effect of introducing direct routes for domestic flights.

The distance flown is smaller, in average $29.4 \mathrm{~nm}$, when flights use direct routes. And the difference is statistically significant. There are more flights with routes of less than 200 $\mathrm{nm}$ when flight use direct routes that when they use airway routes. But all the other route distances are less frequent in the case of direct routes than in the case of airways. This reduction in the distance flown results in savings of fuel and time. Airlines and the environment benefit from such a reduction.

Sector congestion is also reduced by using direct routes instead of airway routes. Controllers spend $32 \%$ of their time managing overloaded sectors when the flights use direct routes, but they spend $35 \%$ when flights use airways.

Peaks of sector congestion are also more separated in time. This reduction might result in safety benefits.

The total number of conflicts detected is reduced about $41.6 \%$ (from 43,894 to 25,638 ) when using direct routes. This results in safety benefits by a reduction of the workload of the controllers.

Ground delays (at the origin airports) increased 24 seconds when using direct routes. There was a limitation in the way FACET uses to assign delays that did not allowed, in this experiment, to measure the airborne or arrival delays. The delays recorded are only due to the GDPs. And the GDPs are using maximum arrival rates for the OEP airports. This does not impose enough restrictions and generates small delays.

Congestion at the destination airports is reduces an average of 0.19 arrivals per 15 minute bin $(95 \%$ confidence interval $[0.17,0.20])$ when using direct routes from TRACON to TRACON. This reduction is statistically significant, but smaller than 1 . So it can be concluded that there is actually no change in the congestion at the destination airport. 


\section{Implications of results}

These results establish an upper bound on the benefits to be derived by Trajectory-based Operations. The result is a win-win scenario for both the airlines and air traffic control. The use of Great Circle Distance routes geographically redistributed the flights reducing workload in the most congested sectors and well as significantly reducing conflicts in flight trajectories. The congestion at the destination airports reduces a small amount, which is a benefit for the airports. It should also be noted that the use of Great Circle Distance routes did not alleviate the flight delays resulting from over-scheduled departure and arrivals.

\section{Future work}

More detailed analysis is required to study the effect at the arrival airports and airspace. Further studies should address questions like, will the use of direct routes produce more noise or pollution in regions not touched by the current system? Future work also includes resolution of several anomalies in the results including: (i) great circle distance routes in excess of the associated flight plan routes, (ii) excessive route distance, (iii) missing flights between scenarios. Adaptive behavior of the airlines will be included in future studies. The adaptive behavior will be based on historical data from previous simulations.

\section{ACKNOWLEDGMENT}

The authors would like to acknowledge the contributions and help from the following persons and institutions.

The research this study is part of is funded by NASA (NRA NNA07CN32A). Furthermore, Natalia Alexandrov, Kapil Sheth, María Consiglio, Brian Baxley, Kurt Neitzke, and Shon Grabbe, all NASA employees, have provided suggestions and comments throughout the whole research process.

From Sensis Corporation, George Hunter and Huina Gao. From George Mason University, Dr. Thomas Speller Jr., Dr. Kenneth De Jong, Dr. Robert Axtell, Dr. George Donohue, Dr. John Shortle, Dr. Rajesh Ganesan, John Ferguson, and Keith Sullivan. From Metron Aviation, Jason Burke, Dr. Terry Thompson, and Norm Fujisaki. From FAA, Joe Post, Kimberly Noonan, and Tony Diana. They have contributed to the improvement of the research.

Finally, thanks to the Ministerio de Ciencia y Tecnología (Minister of Science and Technology) of Costa Rica.

\section{REFERENCES}

[1] JPDO, Concept of Operations for the Next Generation Air Transportation System, Version 2.0. Washington DC, USA: Joint Planning and Development Office, June 2007.

[2] G. Hayman. (2009, June) Trajectory based operations. [Online]. Available: http://www.afceaboston.com/documents/events/cnsatm2009/ Briefings/Tuesday\%20Briefs/Tuesday\%20Afternoon/Track\%202/2\% 20TBO\%20PresentationGeneHayman:pdf

[3] G. Calderon-Meza and L. Sherry, "Establishing an upper-bound for the benefits of nextgen trajectory-based operations," in Electronic Procedings of the 4th International Conference on Research in Air Transportation (ICRAT 2010), June 2010.

[4] A. Barnett, "Free-flight and en route air safety: A first-order analysis," in Operations Research. INFORMS, Nov-Dec 2000, vol. 48, no. 6, pp. 833-845. [Online]. Available: http://www.jstor.org/stable/222992
[5] A. Agogino and K. Tumer, "Regulating air traffic flow with coupled agents," in Proceedings of 7th Int. Conf. on Autonomous Agents and Multiagent Systems (AAMAS 2008), M. Padgham, Parkes and Parsons, Eds., Estoril, Portugal, May 2008, pp. 535-542.

[6] S. Magill, "Effect of direct routing on air traffic control capacity," in Air Transportation Systems Engineering, G. L. Donohue and A. G. Zellweger, Eds., vol. 193. USA: American Institute of Aeronautics ans Astronautics, 2001, pp. 385-396, iSBN 1-56347-474-3.

[7] K. Bilimoria, B. Sridhar, G. B. Chatterji, K. Sheth, and S. Grabbe, "Facet: Future atm concepts evaluation tool," Air Traffic Control Quarterly, vol. 9 no. 1, pp. 1-20, 2001.

[8] B. Sridhar, G. B. Chatterji, S. Grabbe, and K. Sheth, "Integration of traffic flow management decisions," American Institute of Aeronautics and Astronautics, 2002.

[9] R. Jakobovits, P. Kopardekar, J. Burke, and R. Hoffman, "Algorithms for managing sector congestion using the airspace restriction planner," ATM. ATM, 2007. 